\title{
The Natural Convective Heat Transfer in a Partially Divided Enclosure: A Study on the Influence of the Source Position
}

\author{
Francesco Corvaro and Massimo Paroncini \\ Dipartimento di Energetica, Università Politecnica delle Marche, Via Brecce Bianche, 60100 Ancona, Italy \\ Correspondence should be addressed to Francesco Corvaro, f.corvaro@univpm.it
}

Received 1 August 2008; Accepted 11 November 2008

Recommended by Shou-Shing Hsieh

\begin{abstract}
The aim of this paper is to analyse the natural convective heat transfer generated by a source with a height of $\zeta$ located in two different positions inside a square enclosure of side $H$. In the first case, $\delta$ is 0.5 of $H$ while in the second case it is 0.4 . The comparison is based both on the evaluation of the local and average Nusselt numbers at different Rayleigh numbers and on the study of the velocity fields at the same Rayleigh numbers in the two different configurations. The experimental analysis was carried out through a holographic interferometry, to study the heat transfer, and through a 2D-PIV system, to analyse the dynamic behaviour of the phenomenon. Finally, for $\delta=0.5$ we compared the experimental results with those obtained through the volume finite software Fluent 6.3.26. In the analysis, it is possible to see that the position of the source influences both the average Nusselt numbers on the hot surfaces and the development of a small bubble on the upper surface.
\end{abstract}

Copyright ( 2009 F. Corvaro and M. Paroncini. This is an open access article distributed under the Creative Commons Attribution License, which permits unrestricted use, distribution, and reproduction in any medium, provided the original work is properly cited.

\section{Introduction}

The natural convection in a square cavity plays a very interesting role in a lot of engineering applications, such as the solar energy system, the cooling of the electronic circuits, the conditioning of the air, and many others; therefore, it is very important for the applied research. The technical literature presents a lot of studies on natural convection in a square cavity, and many of them analyse the convective phenomenon thanks to numerical simulation.

The work by Ramos and Milanez [1] analysed the natural convection in cavities heated from below by a thermal source, which dissipated energy at a constant rate. Ntibarufata et al. [2] analysed numerically the natural convection in partitioned enclosures with a localized heating from below. Valencia and Frederick [3] elaborated a numerical investigation on the heat transfer of air in square cavities with partially active vertical walls. Aydin and Yang [4] simulated numerically the natural convective heat transfer in air in a square cavity cooled from the sidewalls and heated by a strip placed in the middle of the bottom wall.

Cesini et al. [5] analysed the convective heat transfer generated by a horizontal cylinder located in a square cavity.
This study was carried out both through an experimental analysis and a numerical study.

In this paper, the cavity testing is a square enclosure, and we analysed the influence of the position of a heated strip on the natural convection heat transfer; the heated strip was placed on the bottom of the cavity.

Two different positions of the hot strip are analysed: $\delta=0.5$ of $H$ (symmetrical position) and $\delta=0.4$ of $H$ (asymmetrical position). The width of the heat strip is $\varepsilon=$ 0.2 of $H$ and the height of the square cavity is $H=0.05 \mathrm{~m}$. The height of the strip is $\zeta=0.5$. The cavity is cooled from the sidewalls at the same time and filled with air $(\mathrm{Pr}=0.71)$. The laminar condition of the airflow was considered both in the numerical study and in the experimental analysis.

The symmetrical configuration was studied both through a holographic interferometer to evaluate its thermal behaviour and through a 2D-PIV system to determine dynamic structures such as the stream functions, the velocity vectors, and the velocity maps. These experimental results were used to validate a numerical model made by a finite volume code (Fluent 6.3.26). The achievement of a good agreement between the experimental data and the numerical ones allows us studying the asymmetrical position through 


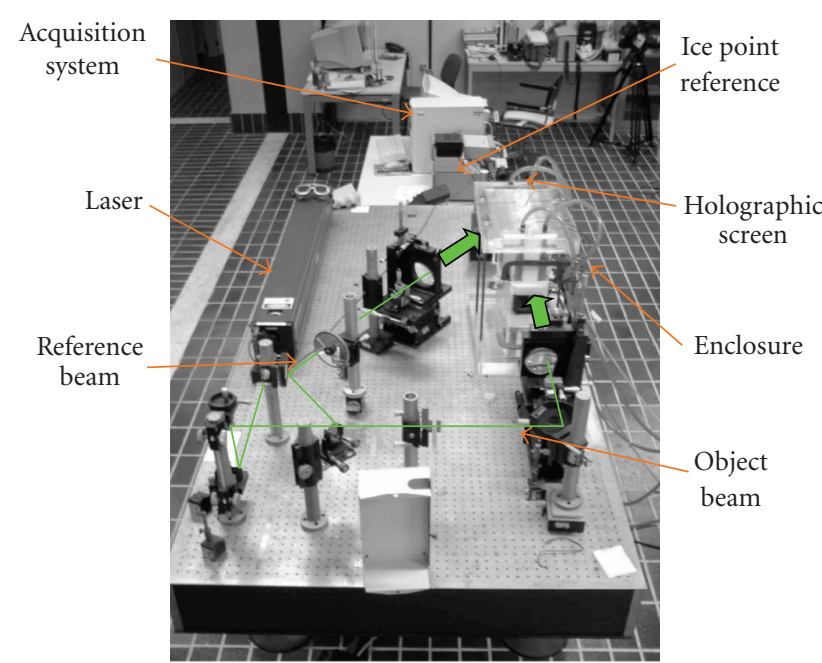

Figure 1: Holographic interferometry system.

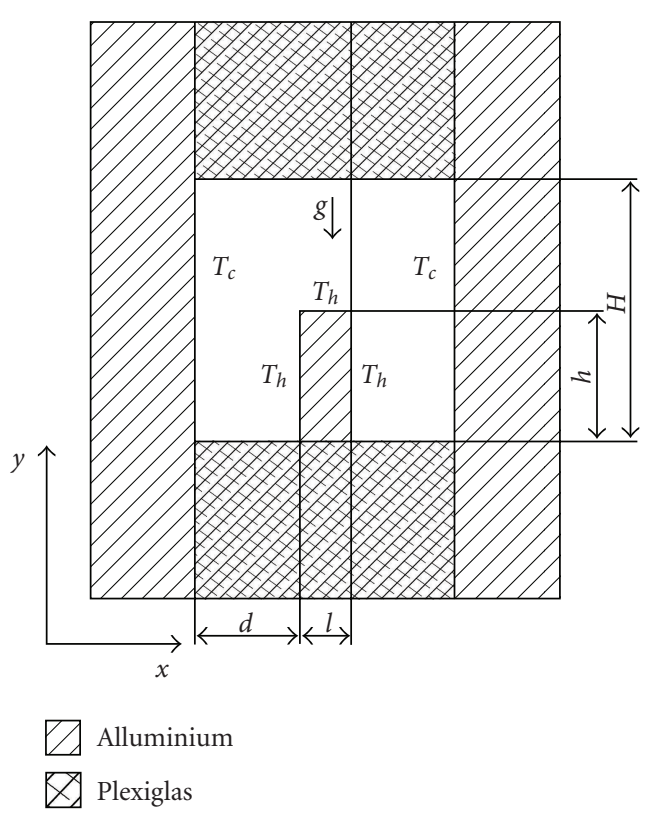

Figure 2: The cavity structure.

the numerical code. In particular, the finite volume software was used to obtain the local and average Nusselt numbers on the hot surfaces. While the dynamical behaviour for $\delta=0.4$ was analysed also through the PIV system.

The experimental research analysed different Rayleigh numbers in particular from $6.5 \cdot 10^{4}$ to $3.2 \cdot 10^{5}$. Moreover, we expanded this range thanks to a numerical study made between $3 \cdot 10^{4}$ and $3.5 \cdot 10^{5}$ after a first comparison between the numerical and experimental results.

\section{Experimental Equipment}

2.1. Holographic Interferometry. The main components of the experimental apparatus, represented in Figure 1, include the test cell, filled with air at atmospheric pressure, the pneumatic auto-levelling table, the holographic interferometer, the optical instruments, three thermostatic baths, and the data acquisition system. A schematic drawing of the test cell is shown in Figure 2 where it is possible to see also the Cartesian coordinate system $(x, y)$.

It has a square transversal section of $0.05 \mathrm{~m}$ side, and it is heated from below. The heat hot source is maintained at a temperature $T_{h}$ by a fluid which circulates through one of the three thermostatic baths. This temperature was changed from one test to another in order to obtain different Rayleigh numbers for each test. The lateral vertical walls (that are made up of aluminium) are kept at a temperature $T_{c}$ by a fluid, which is cooled by two other thermostatic baths. This temperature was set at $291.16 \mathrm{~K}$ and was maintained constant during each test. The top and the bottom surfaces of the enclosure are made up of Plexiglas in order to neglect the heat leakage through these walls. Anyway, it is not feasible in the experimental analysis. So, the boundary condition at these surfaces has been implemented in order to take into account the conductive heat flux through the Plexiglas walls. The end vertical walls are made up of glass to guarantee the access to the laser beam.

The dimension of the test cell along the longitudinal direction is $0.42 \mathrm{~m}$, so it is possible to neglect the end effects and to consider the problem as a two-dimensional one.

The temperature on the heat source and on the vertical walls is measured through copper-constantan thermocouples that are located $1 \mathrm{~mm}$ under the surface of the test volume. Three of them are put on the hot source, and the other ten thermocouples are set on the vertical walls. The last one is positioned in the middle of the air region, and it gives us a reference temperature to analyse the interferograms. The difference between the three values of the temperature on the hot plate is about $0.2 \mathrm{~K}$, consequently it is possible to consider an isotherm situation; on the lateral wall the maximum difference between the five thermocouples is $0.4 \mathrm{~K}$.

The light source is an argon-ion laser with a nominal power rating of $4 \mathrm{~W}$, with etalon for the $514.5 \mathrm{~nm}$ wavelength. Both the object and the reference beams have a maximum diameter of $0.15 \mathrm{~m}$.

The optical setup allows us the use of the doubleexposure or of the real-time holographic interferometry techniques, for the steady-state and the temporal evolution measurements of heat transfer processes. The real-time technique has also been used to check the presence of plume oscillations.

2.2. 2D-PIV System. The test cell, the acquisition system, and the thermostatic elements are the same used for the holographic setup.

The particle image velocimetry (PIV) is finalized to make instantaneous velocity measurements in the crosssection of a flow. The PIV system provides the velocity fields with the distance made by the particles-seeded in the flow. The seeding particles nature must be chosen in order to provide a real description of the airflow. For a more 


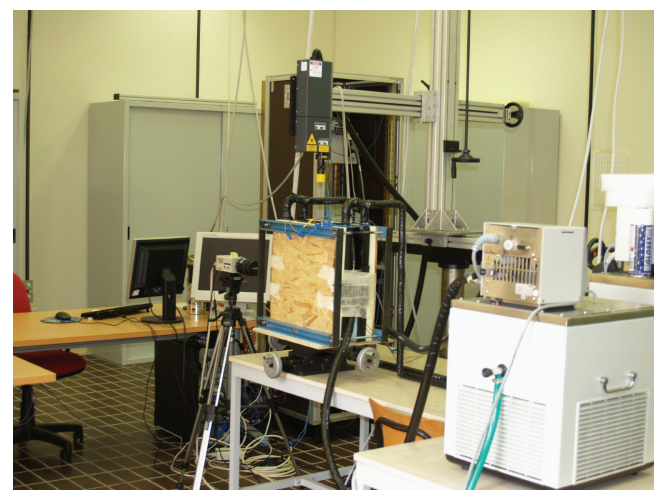

(a)

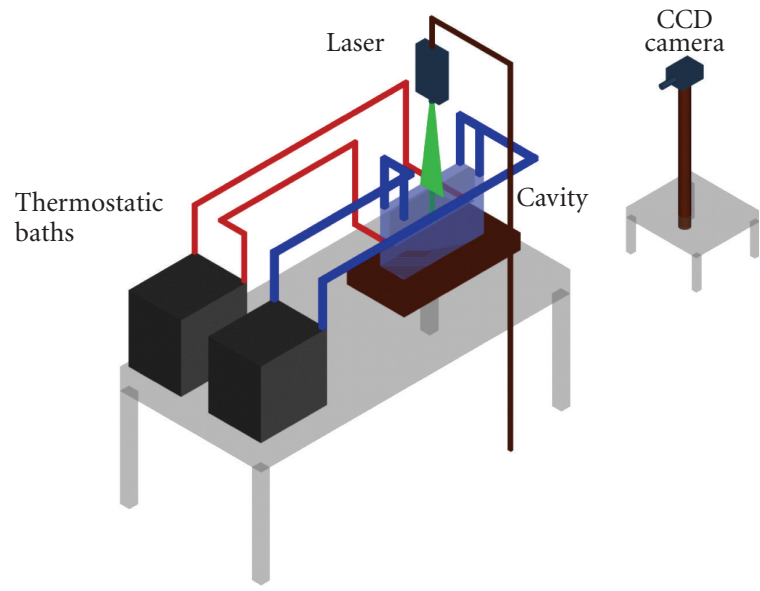

(b)

Figure 3: 2D-PIV system.

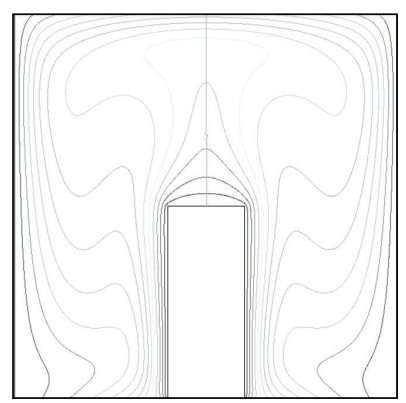

(a)

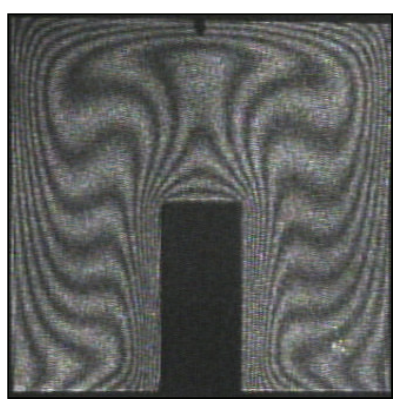

(b)
Figure 4: Comparison between numerical and experimental isothermal lines at $\mathrm{Ra}=2 \cdot 10^{5}$ for $\delta=0.5$.

detailed explanation of the PIV technique, it is possible to see Raffel et al. [6].

In these experiments, oil particles were used and they were nebulised by an air compressor system; the diameter of the particle was about $1 \mu \mathrm{m}$. The laser used is a double cavity $\mathrm{Nd}$-Yag laser with a wavelength of $532 \mathrm{~nm}$.

The data analysis was performed using the software package "Dynamic Studio," provided by Dantec Dynamics, Bristol, UK (2006) [7].

The images of the area of the flow field, lighted by a light sheet, are captured by a CCD camera (Hamamatsu camera C8484-05C with $1344 \times 1024$ number of pixels). The camera lent was covered by a filter with a wavelength $\lambda=532 \mathrm{~nm}$ in order to record only the direct or scattered light from the laser source inside the PIV cavity.

The light sheet gets in through the upper part of the enclosure made up of Plexiglas, and it lights up a crosssection of the cavity in the middle of the cell. The camera, that is located perpendicularly to the light sheet, captures the images, which are sent to a computer to be processed.

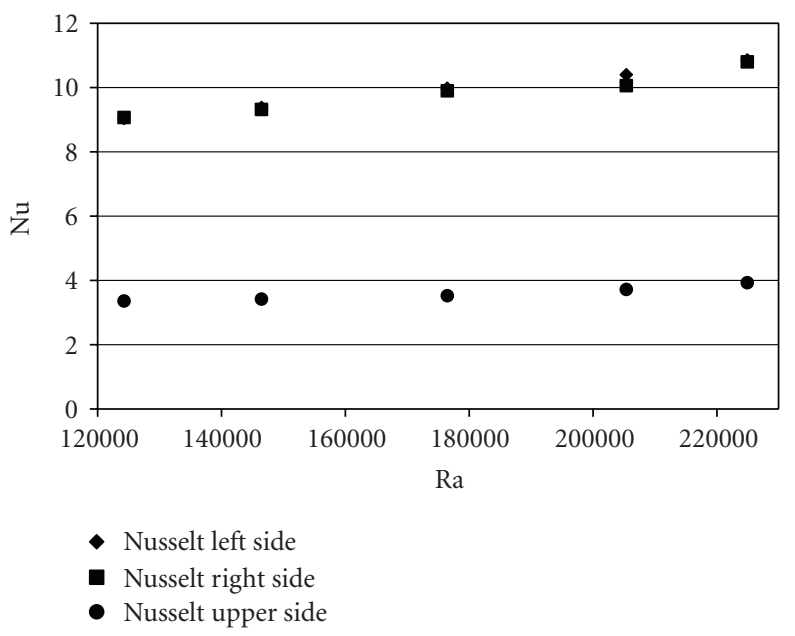

Figure 5: Average Nusselt numbers as function of Rayleigh numbers on the hot surfaces for $\delta=0.5$.

A small pane of glass to permit an optical access to the camera covers the front sidewall of the cavity; instead the rear side is rigged with a $0.05 \mathrm{~m}$ square piece of wood which has an opening in the middle. Through it, we sprinkle the oil particles for 20 seconds before the beginning of each test. The top and the bottom surfaces of the enclosure are made up of Plexiglas with a thickness of $0.03 \mathrm{~m}$ to neglect the leaks of heat through these surfaces. The PIV equipment is shown in Figure 3. The Rayleigh numbers analysed change from $6.5 \cdot 10^{4}$ to $3.2 \cdot 10^{5}$.

The processing technique used in this study is Dantec adaptive-cross correlation, described by Dantec Dynamics (2006) [7], with an interrogation area of $32 \times 32$ pixels.

The overlap of the interrogation area in the horizontal and vertical directions is $50 \%$. Furthermore, a moving average validation is applied; this method validated vectors based on comparison between neighbouring vectors: an averaging 


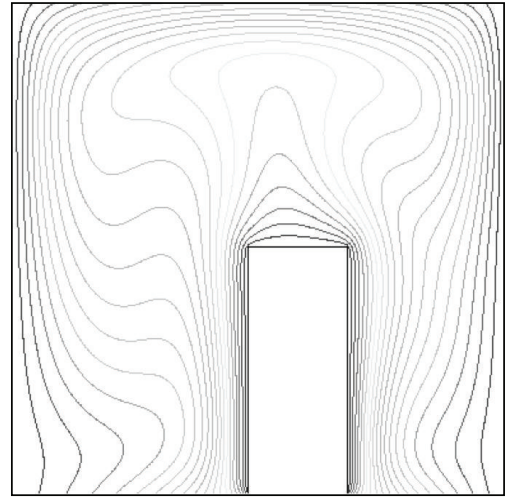

FIGURE 6: Numerical isothermal lines for $\delta=0.4$.

area of $3 \times 3$ is used with an acceptance factor of 0.1 and there are 3 iterations.

This method substituted outliers due to false correlations resulting from reflections at the cell walls and from the other invalid vectors.

During our tests, we evaluated also the repeatability of the experiment. We repeated the same test with the same initial boundary condition at different Rayleigh numbers for each geometrical configuration. Then after 45 minutes from the beginning, we stored the modulus of the velocity vector at different heights: in particular at $16,1 \mathrm{~mm}, 26,1 \mathrm{~mm}$, and $36,1 \mathrm{~mm}$ from the floor of the enclosure. During these checks, the same velocity vector values were obtained. There is only a small difference between the three tests near the left side of the cavity. This fact is due to the reflections of the laser light near the contours of the enclosure that create some noise in the PIV image, and so there is a lack of precision in the velocity vectors.

Similar results are obtained in each test that is made to determinate the repeatability of our experiments.

\section{Numerical Procedure and Setting}

The Fluent solution methods are known in the scientific background. Particularly, the numerical simulation is developed through the finite volume code Fluent 6.3.26 using the Boussinesq approximation for the air and a two-dimensional model.

The numerical results are carried out with segregated solvers [8] for $3 \cdot 10^{4}<\mathrm{Ra}<3.5 \cdot 10^{5}$. Thanks to this approach; the governing equations are solved sequentially and segregated from one another. According to this method, each discrete equation is linearized implicitly with respect to that equation's dependent variable.

As for the spatial discretization, in our tests a secondorder up-wind implicit scheme is employed for the conservation equations; the pressure interpolation is provided with the body-force-weighted scheme, and the pressure-velocity coupling is achieved using the simple algorithm.

The diffusion terms are central differenced with a secondorder accuracy.
In the performed analysis, the cavity was reproduced with the real dimensions, and the temperature of the lateral walls and of the heated strip is assigned. In the Fluent model, we introduced also the Plexiglas elements used to make the upper and the lower surfaces of the experimental enclosure. It was necessary to simulate the conductive heat transfer between these elements and the cold wall.

A mesh structure with quadrilateral cell elements is performed. A study of the mesh was carried out preliminarily in order to obtain the lowest number of cells necessary to perform an analysis with results that are independent from the choice of the cell number.

At this step, we noticed that a good mesh was necessary to solve important dynamic structures. Particularly, thanks to the PIV system; we saw very small vortexes on the upper surface of the hot strip with $\zeta=0.5$. To evaluate them, we used a mesh of 73000 cells in the enclosure area with a boundary layer on every hot surfaces of the strip. According to these conditions, the variables used to compare the numerical results and the experimental ones (as the local and average Nusselt numbers or the heat flux) showed an independent behaviour respect the grid size.

During the analysis, we used the steady procedure and the admitted value for all residuals to obtain a convergence that was $10^{-6}$, for the equations of continuity and for the momentum, while it was lower to $10^{-12}$ for the equation of energy.

The local and average Nusselt numbers on the hot surfaces, the velocity fields, the stream functions, and the velocity vectors distribution inside the cavity obtained numerically were compared to the experimental results.

\section{Results and Discussion}

The real-time interferograms are obtained with a finitefringe field, while an infinite-fringe field is used in the double exposure interferograms; so doing the fringe pattern shows directly the distribution of the isothermal lines.

The evaluation of the interferograms is achieved thanks to the help of a travelling microscope to obtain the intensity distribution. Density and temperature distribution are obtained by the usual methods of inversion [9]. According to Hauf and Grigull [9], the expected accuracy of a small fringe number (less than 30) can be of about 10\%.

The study deals with the effects of the position of the heat source; its length is $1 / 5$ of the side of the enclosure, and the height is $\zeta=0.5$ of $H$. Two positions are analysed: $\delta=0.5$ of $H$ (symmetrical position) and $\delta=0.4$ of $H$ (asymmetrical position). The experimental Rayleigh numbers investigated through the holographic interferometry vary from about $1.2 \cdot 10^{5}$ to $2.3 \cdot 10^{5}$, while through the PIV system this range changed between $6.5 \cdot 10^{4}$ and $3.2 \cdot 10^{5}$.

The symmetrical position was studied thanks to the PIV system and the holographic interferometry while the asymmetrical one was analysed thanks to the 2D PIV and through the numerical code to determinate the heat transfer on the hot surfaces. The numerical code was validated with the comparison between the numerical and experimental Nusselt numbers for $\delta=0.5$. 


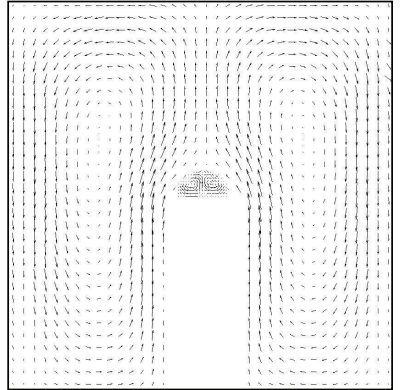

$\mathrm{Ra}=1.78 \times 10^{5}$

(a)

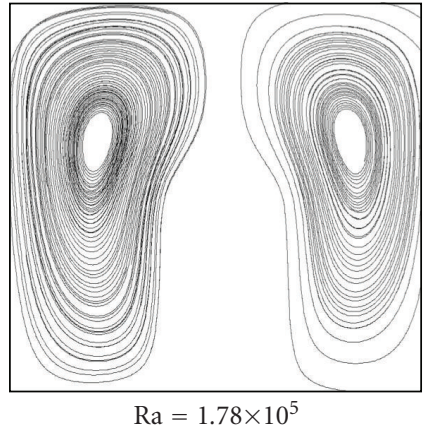

(b)

FIGURE 7: Velocity vectors and streamfunctions for the symmetrical configuration obtained through PIV.

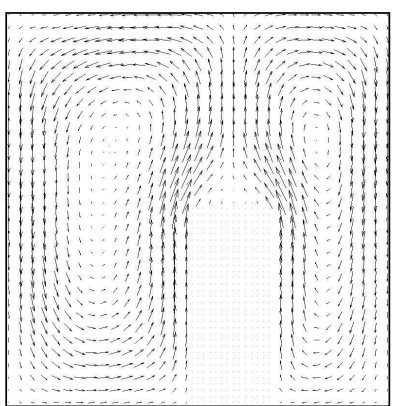

$\mathrm{Ra}=1.78 \times 10^{5}$

(a)

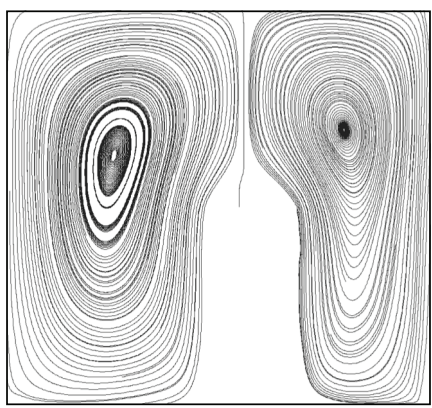

$\mathrm{Ra}=1.78 \times 10^{5}$

(b)

FIGURE 8: Velocity vectors and streamfunctions for the asymmetrical configuration obtained through PIV.

The dimensionless parameters used are

$$
\begin{array}{rlrl}
X & =\frac{x}{H}, & Y=\frac{y}{H}, & \theta=\frac{T-T_{c}}{T_{h}-T_{c}}, \\
\varepsilon=\frac{l}{H}, & \zeta=\frac{h}{H}, & \delta=\frac{d+l / 2}{H} .
\end{array}
$$

In both two cases, the hot strip is located on the bottom of the enclosure with a distance from the sidewalls of $\delta=0.5$ (symmetrical position).

The local Nusselt number, $\mathrm{Nu}(X)$, is calculated on the heat source with the expression

$$
\mathrm{Nu}_{h}=-\left.\frac{\partial \theta}{\partial Y}\right|_{Y=0}
$$

for the upper surface of the strip. While for the lateral side of the source with $\zeta=0.5$, we use

$$
\mathrm{Nu}_{h}=-\left.\frac{\partial \theta}{\partial X}\right|_{X=0} .
$$

The average Nusselt number, $\overline{\mathrm{Nu}}$, on the heat source is given for the upper surface of the source by

$$
\overline{\mathrm{Nu}_{h}}=\frac{1}{\varepsilon} \cdot \int_{0}^{\varepsilon} \mathrm{Nu}(X) d X .
$$

While for the lateral surfaces, we used the following expression:

$$
\overline{\mathrm{Nu}_{h}}=\frac{1}{\zeta} \cdot \int_{0}^{\zeta} \mathrm{Nu}(Y) d Y .
$$

Analysing the interferograms obtained through the double exposure technique, the local and the average Nusselt numbers on the hot surface for $\delta=0.5$ were evaluated. This step was carried out at different Rayleigh numbers. The result of this procedure is shown in Figure 5. It is possible to see that the growing of the Rayleigh number creates a progressive development of the natural convective heat transfer indicated through the growing of the Nusselt number.

In the second column of Figure 4, it is possible to note an example of a double exposure interferograms. The black and white lines are comparable to the distribution of the isothermal lines in the cavity. Then in the second column, a similar map obtained through the numerical code is shown. It is already possible to see a good qualitative agreement between the numerical and the experimental temperature distributions in the enclosure.

By studying in depth the image of Figure 4, it is also possible to note a high number of lines near the lateral surfaces; this indicates that in these zones a better natural convective heat transfer is developed. This assertion is 
TABLE 1: Comparison between average experimental Nusselt numbers and numerical ones on the lateral sides for $\delta=0.5$.

\begin{tabular}{lllcllc}
\hline $\mathrm{Ra}$ & $\begin{array}{l}\text { Nu rg } \\
\text { exp }\end{array}$ & $\begin{array}{l}\text { Nu rg } \\
\text { num }\end{array}$ & $\Delta \%$ & $\begin{array}{l}\text { Nu lf } \\
\text { exp }\end{array}$ & $\begin{array}{l}\text { Nu rg } \\
\text { num }\end{array}$ & $\Delta \%$ \\
\hline $1.24 E+05$ & 9.04 & 8.98 & 0.61 & 9.07 & 8.98 & 1.00 \\
$1.46 E+05$ & 9.38 & 9.40 & -0.19 & 9.32 & 9.39 & -0.78 \\
$1.76 E+05$ & 9.98 & 9.89 & 0.90 & 9.9 & 9.89 & 0.14 \\
$2.05 E+05$ & 10.4 & 10.31 & 0.87 & 10.41 & 10.31 & 0.87 \\
$2.25 E+05$ & 10.86 & 10.57 & 2.68 & 10.8 & 10.57 & 2.16 \\
\hline
\end{tabular}

TABle 2: Comparison between average experimental Nusselt numbers and numerical ones on the superior side for $\delta=0.5$.

\begin{tabular}{lccc}
\hline $\mathrm{Ra}$ & Nu up exp & Nu up num & $\Delta \%$ \\
\hline $1.24 E+05$ & 3.36 & 3.67 & -9.29 \\
$1.46 E+05$ & 3.42 & 3.74 & -9.43 \\
$1.76 E+05$ & 3.525 & 3.82 & -8.47 \\
$2.05 E+05$ & 3.72 & 3.89 & -4.60 \\
$2.25 E+05$ & 3.93 & 3.93 & -0.05 \\
\hline
\end{tabular}

TABLe 3: Average numerical Nusselt numbers for $\delta=0.4$.

\begin{tabular}{lccc}
\hline $\mathrm{Ra}$ & Nu rg & Nu lf & Nu up \\
\hline $1.24 E+05$ & 8.98 & 9.02 & 4.05 \\
$1.46 E+05$ & 9.41 & 9.45 & 4.10 \\
$1.76 E+05$ & 9.91 & 9.97 & 4.16 \\
$2.05 E+05$ & 10.34 & 10.42 & 4.20 \\
$2.25 E+05$ & 10.61 & 10.69 & 4.23 \\
\hline
\end{tabular}

TABLE 4: Coefficients for the correlationship number 5.

\begin{tabular}{lcccc}
\hline & $a$ & $b$ & $\Delta_{\max }$ & $R^{2}$ \\
\hline Nu up exp $(\delta=0.5)$ & 0.1714 & 0.2523 & $-2.47 \%$ & 0.91 \\
Nu up num $(\delta=0.5)$ & 0.9496 & 0.1153 & $-0.92 \%$ & 0.99 \\
Nu up num $(\delta=0.4)$ & 1.7525 & 0.0715 & $3.60 \%$ & 0.97 \\
Nu rg exp $(\delta=0.5)$ & 0.2488 & 0.3057 & $0.89 \%$ & 0.99 \\
Nu rg num $(\delta=0.5)$ & 0.3624 & 0.2737 & $0.45 \%$ & 0.99 \\
Nu rg num $(\delta=0.4)$ & 0.3338 & 0.2807 & $1.29 \%$ & 0.99 \\
Nu lf $\exp (\delta=0.5)$ & 0.3668 & 0.2727 & $2.17 \%$ & 0.93 \\
Nu lf num $(\delta=0.5)$ & 0.3592 & 0.2744 & $0.56 \%$ & 0.99 \\
Nu lf num $(\delta=0.4)$ & 0.3082 & 0.2878 & $0.31 \%$ & 0.99 \\
\hline
\end{tabular}

confirmed by Figure 5 where the Nusselt numbers on this surface are quite high. In this figure, it is also possible to note the difference between the Nusselt numbers on the lateral sides and the upper surface. There is an important reduction in the efficiency of the heat transfer between the lateral and the superior surfaces. In fact, by analysing Figure 4 , it is possible to find very few fringes on the upper side of the source. This behaviour is also confirmed by the numerical code, and it is probably connected to two vortical structures, which are generated by the air in this zone. These particular elements were visualised through the PIV equipment and described in the following sections in details.

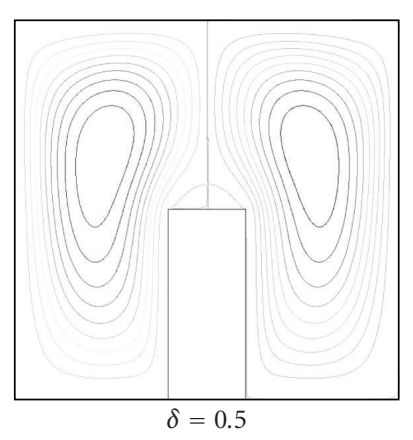

(a)

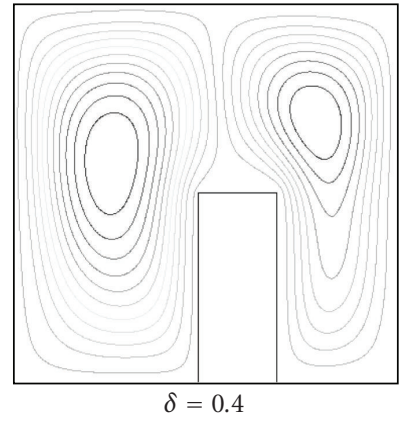

(b)
Figure 9: Numerical stream functions.

Moreover, at this step of the study, the agreement between the numerical Nusselt numbers and the experimental ones obtained for the symmetrical position $(\delta=0.5)$ is analysed. In Tables 1 and 2, the average Nusselt numbers on the hot surfaces are shown. It is possible to note (see Table 1) that for the lateral surfaces the percentage difference between the experimental value and the numerical one is lower than $3 \%$. This fact points out a very good agreement between the experimental procedure and the numerical analysis. By analysing Table 2, this difference grows up even if it remains under $10 \%$. This element is connected to the characteristics of the interferograms. If we analyse Figure 4(b), we will note that there are very few fringes on the upper surface. For this reason, the interpretation of the interferometric image is more difficult; consequently, we obtain a lower accuracy; but even in this case, it is possible to conclude that the numerical model approaches in a satisfactory way the real system.

After this important validation, the numerical code was used to simulate the natural convective heat transfer for the asymmetrical position $(\delta=0.4)$. The results of this procedure are shown in Table 3 and in Figure 6. In the table, it is possible to see the average Nusselt numbers. Analysing these values, a small loss of balance among the lateral surfaces is evident. On the left side, the average Nusselt numbers begin to become bigger than the ones on the right side. In Figure 6, it is possible to see the isothermal lines. Analysing this image, it is evident that the right side is nearer to the cold wall than the other one. Through the PIV analysis, it is possible to note that on this zone, the airflow is constricted to a smaller part of the enclosure differently from the air in the left zone. It creates a small deceleration in the velocity field; consequently, we find a worse natural convective heat transfer on this side.

Moreover, it is possible to find another difference between the two positions on the upper surface in the development of the natural convective heat transfer. For $\delta$ $=0.4$, the average Nusselt number on this side grows up in comparison with the other configuration. This means that the natural convective heat transfer improves its effect. This element could be connected to the evolution of the vortical structures visualized through the PIV system and analysed in the following section. 


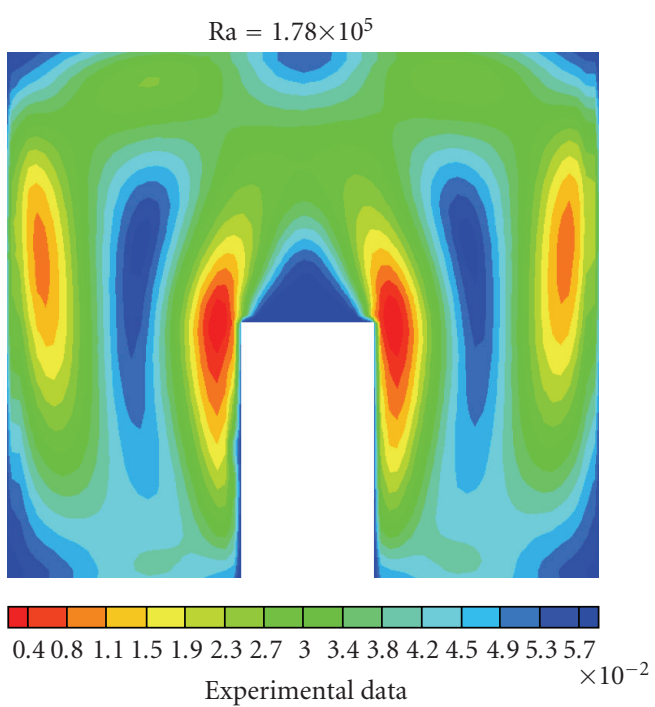

(a)

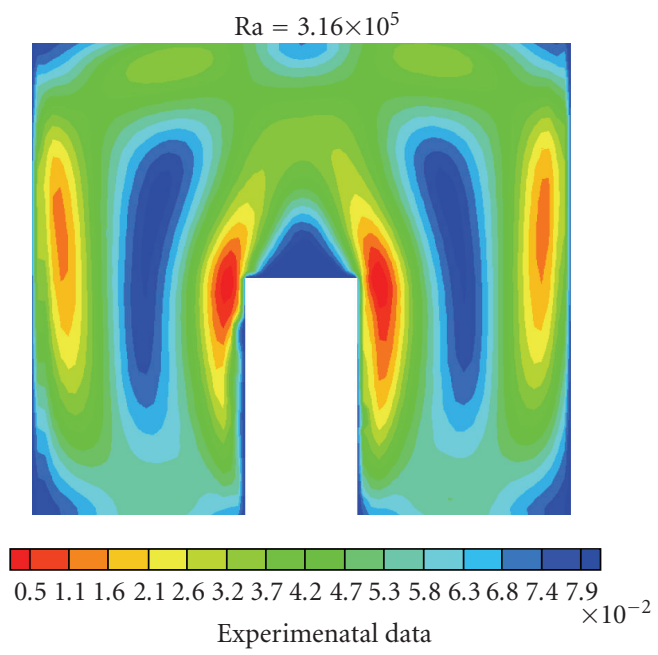

(c)

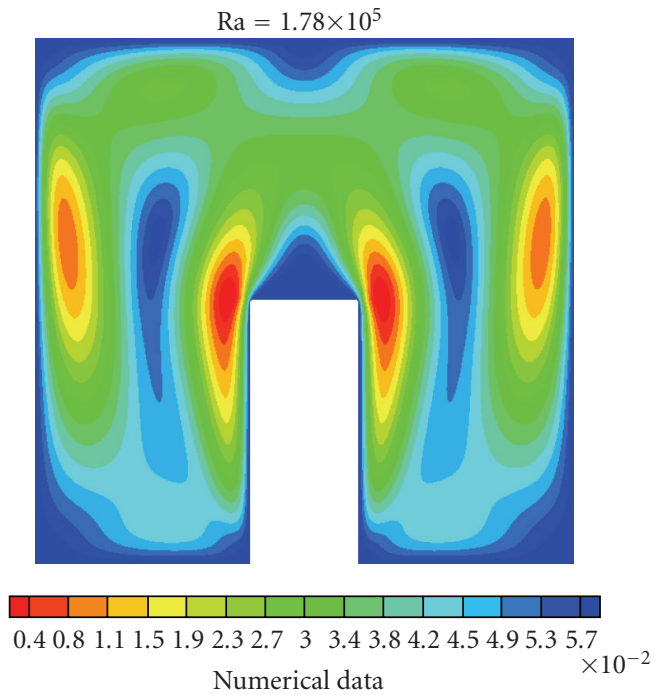

(b)

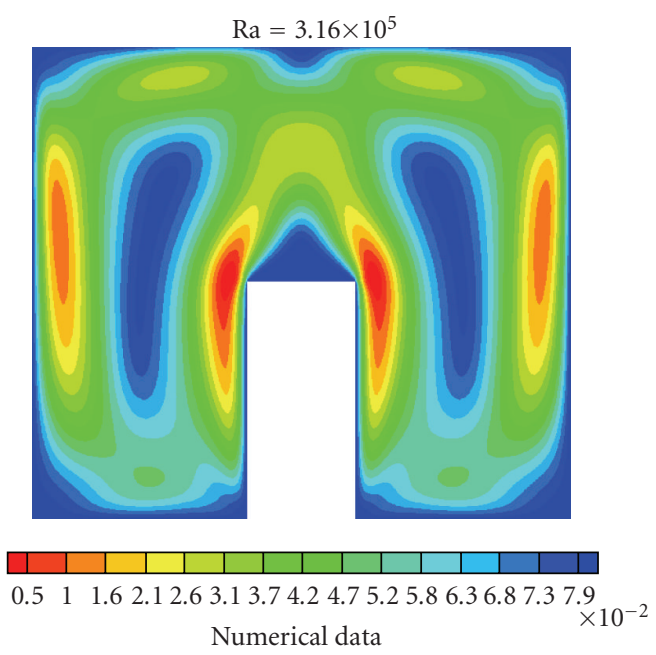

(d)

Figure 10: Velocity maps in $\mathrm{m} / \mathrm{s}$ for $\delta=0.5$.

Finally, the numerical and experimental relationships between the Rayleigh numbers and the average Nusselt numbers were evaluated. The correlation is

$$
\overline{\mathrm{Nu}}=a \cdot \mathrm{Ra}^{b},
$$

where $a$ and $b$ are shown in Table 4 . In this table, it is also possible to see the biggest percentage deviation and the $R^{2}$ for each correlation used. $R^{2}$ is an indicator that shows the agreement between the esteemed value of the empirical function and the real one. The more $R^{2}$ is close to 1 , the more the relationship can be considered a good real data approximation.

In the following step of the study, the 2D-PIV system was used to analyse the dynamic structures connected to the natural convective heat transfer in these two geometrical configurations. The first results are shown in Figures 7 and 8. It is possible to note the presence of two big convective counter-rotating cylinders which begin to move when the thermostatic fluids enter in the aluminium plates. They are visible in each position analysed even in the numerical analysis as shown in Figure 9. Analysing the asymmetrical position $(\delta=0.4)$, it is possible to note that these two cylinders do not develop symmetrically. Moreover, Figure 7(a) shows the position of two other small vortical structures on the upper surface that are very important for the natural convective phenomenon.

Through these images, it is also possible to understand why the Nusselt numbers on the left side of the source for $\delta=0.4$ are slightly bigger than the ones in the other position. Figure 8 (b) shows a bigger vortex on the left side in the asymmetrical configuration. This fact indicates that the airflow can create a more efficient circulation in this zone. By analysing the velocity maps, it is possible to see that the highest velocity values are concentrated in this area. For this 


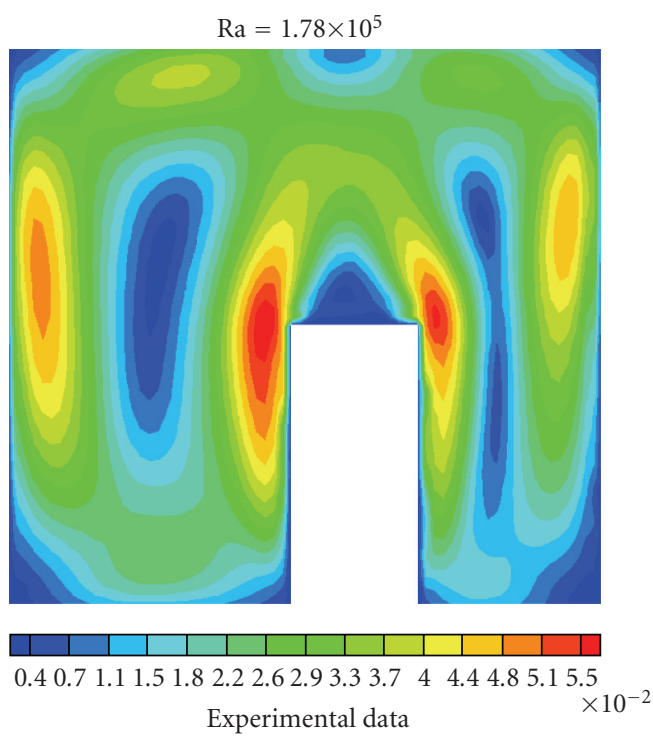

(a)

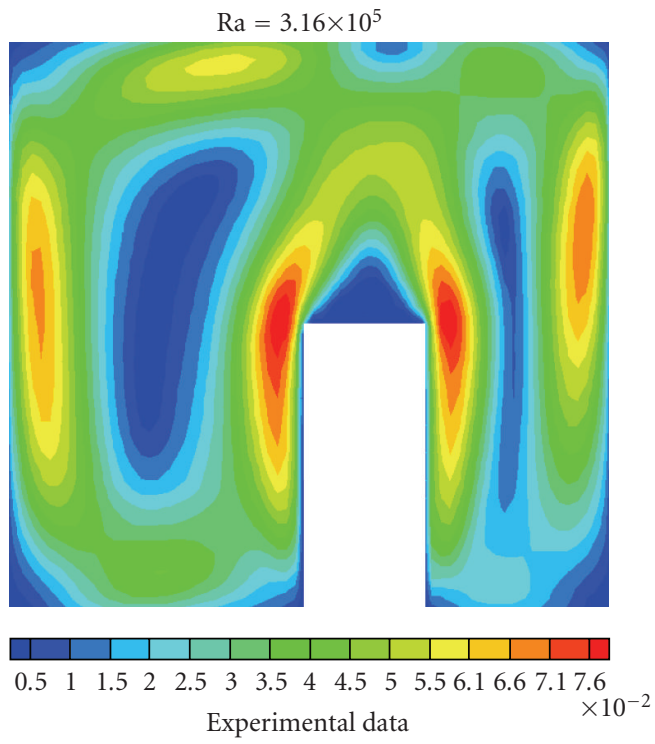

(c)

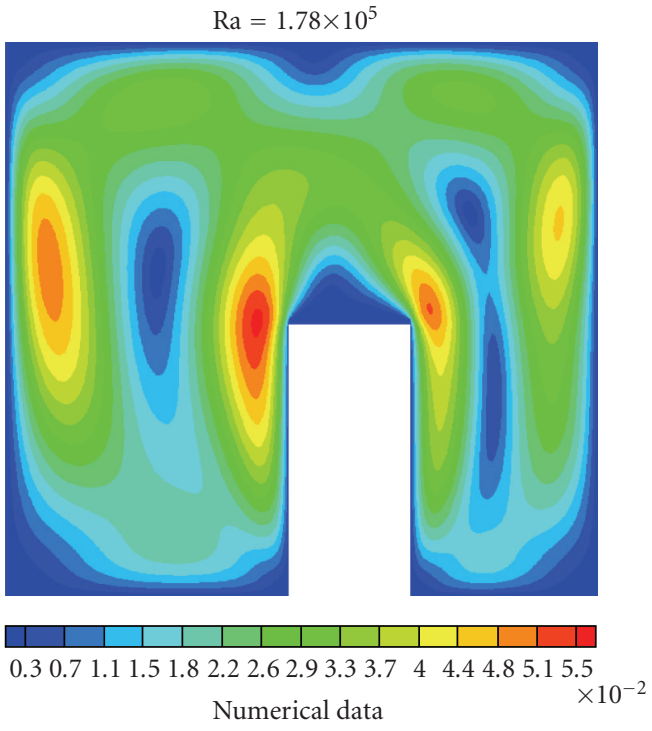

(b)

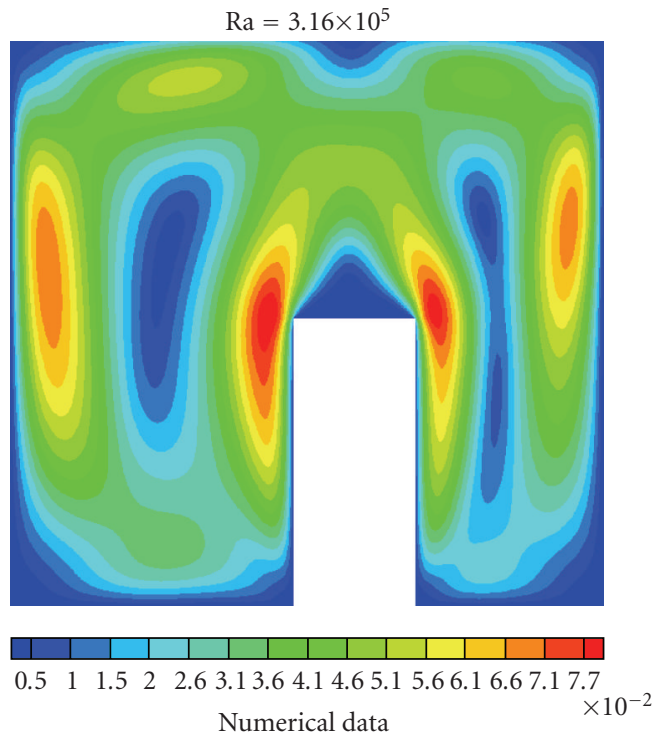

(d)

FIGURE 11: Velocity maps for $\delta=0.4$.

reason, it is possible to find a better natural convective heat transfer on the left side of the source than the one on the other side.

During each test, it was possible to see that the dynamic structures (distribution of the velocity vectors and streamfunctions) did not change with the Rayleigh number. Instead, the modulus of the velocity vectors grows up according to the growth of the Rayleigh number.

The velocity maps are shown in Figures 10 and 11. In the first column, it is possible to see the experimental results while in the second column there are the numerical ones. Comparing these data, a good agreement between the experimental values and the numerical code is evident as the velocity value distribution inside the enclosure is comparable both to the velocity peak and to the average velocity value.

Finally, the vortical structures, shown in Figure 7(a), on the upper surface were analysed. Their rotation axis is parallel to the longitudinal direction of the cavity $(z$ axis), and they have the same behaviour both in the experimental analysis and in the numerical study. In particular, by analysing Figure 12, it is possible to note that they begin to develop at high Rayleigh numbers (first column) while at low Rayleigh numbers the airflow has a simple vertical development (second column). These small vortexes influence the natural convective heat transfer on the upper side. 

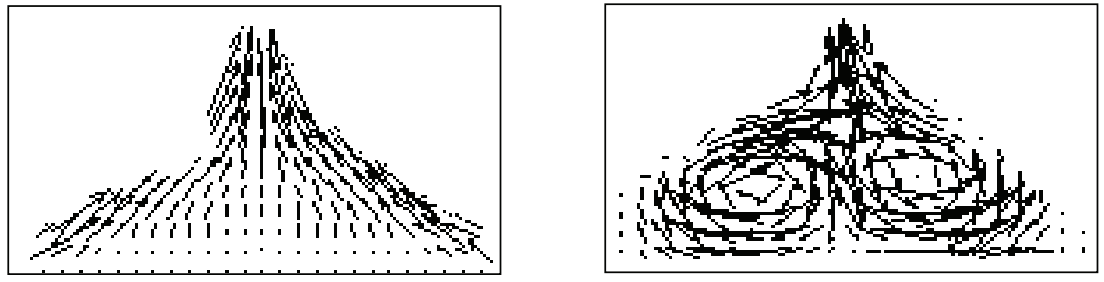

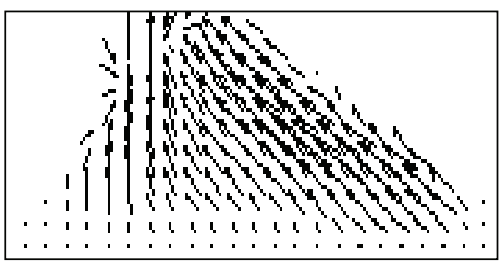

(a) $\mathrm{Ra}=6.39 \cdot 10^{4}$

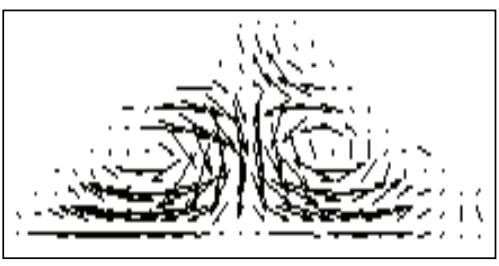

(b) $\mathrm{Ra}=1.78 \cdot 10^{4}$

Figure 12: A particular of the vortical structures on the upper surface of the strip for $\delta=0.5$ (first row) and for $\delta=0.4$ (second row).

For $\delta=0.5$ (first row of Figure 12), they have a symmetrical development. The big cylinders keep the air in this small zone; consequently, the flow slows down. This creates a worst heat transfer as confirmed by analysing the average Nusselt numbers during the description of the holographic interferometry results. For $\delta=0.4$ (second row of Figure 12), the two small counter-rotating cylinders have not perfect symmetrical structures. This change allows the two small counter-rotating cylinders to be connected to the big dynamic cylinders. So making the small bubble on the upper surfaces has the possibility to have a more efficient change of air. Consequently, the velocity values are bigger than the ones of the symmetrical configuration; so the heat transfer is better as described by analysing the growth of the average Nusselt numbers on the upper side for $\delta=0.4$.

\section{Conclusion}

In this paper, the influence of the position of a heat source on the natural convective heat transfer in a square cavity of side $H$ was analysed. The source has a height $\zeta=0.5$ of $H$, and the enclosure is filled with air $(\operatorname{Pr}=0.71)$.

The analysis was conducted experimentally both through the holographic interferometry and through the particle image velocimetry for Rayleigh number from $6.5 \cdot 10^{4}$ to $3.2 \cdot 10^{5}$. While we expanded this range with a numerical study between $3 \cdot 10^{4}$ and $3.5 \cdot 10^{5}$, the software used was a finite volume code, Fluent 6.3.26. The PIV analysis evaluated the flow structures connected to the convective phenomenon like the velocity fields, the streamfunctions and the velocity vector distributions while, thanks to the holographic interferometry, the average and local Nusselt numbers were calculated.

Two different positions of the source were analysed: the symmetrical position and an asymmetrical one. In the first case, the distance between the right side of the strip and the right side of the cavity is $\delta=0.5$ of $H$; in the second case this distance is $\delta=0.4$ of $H$. The thickness of the heat strip is $\varepsilon=$ 0.2 of $H$.

Through the holographic interferometry, the heat transfer was analysed. In particular, the local and average Nusselt numbers on the hot surfaces were evaluated. The natural convection is more efficient on the lateral sides of the strip. On the upper surface, the average Nusselt numbers at the same Rayleigh numbers are lower than on the lateral ones. This is confirmed by the numerical code and is connected to two particular vortical structures analysed through the PIV system. Then through the experimental data, the numerical code was validated and used to analyse the asymmetrical configuration. Two important differences are evident making a comparison with the symmetrical position. There is a small difference between the average Nusselt number on the right side and the one on the left side at the same Rayleigh number. In particular, the left surface has a higher Nusselt number than the other one. It is possible to understand this event by analysing the velocity maps obtained through the PIV system. In the left zone of the enclosure for $\delta=0.4$, the vortex is bigger than the one on the other side. For this reason, a bigger high-velocity zone is present near the left side of the source. Consequently, the natural convection of air is more efficient.

The second difference is about the average Nusselt numbers on the upper surface. In the asymmetrical configuration, their values grow up in comparison to the symmetrical position. This fact deals with the behaviour of the two small cylinders on this surface. For $\delta=0.5$, they have a symmetrical development that creates an isolation surface between these small vortical structures and the big convective cylinders. Consequently, the velocity field in this zone slows down, and the natural convective heat transfer is reduced. Instead for $\delta=0.4$, their symmetrical characteristic begins to have a lower influence, so the air in this confined zone can be connected to the flow of the big vortexes. Consequently, the velocity field is quicker than the one analysed in the first 
geometrical configuration. In this way, the natural convective heat transfer grows up as indicated by the growing of the average Nusselt numbers.

\section{Nomenclature}

$g$ : $\quad$ Modulus of the gravity vector $\left(g=9.81 \mathrm{~m} \mathrm{~s}^{-2}\right)$

$\Delta t: \quad$ Time between two laser pulses $(\mu \mathrm{s})$

$H: \quad$ Square cavity side $(\mathrm{m})$

$k$ : $\quad$ Thermal conductivity $\left(\mathrm{Wm}^{-1} \mathrm{~K}^{-1}\right)$

$l: \quad$ Heat source length $(\mathrm{m})$

$h$ : Heat source height $(\mathrm{m})$

$d$ : Distance of the hot strip from thesidewall (m)

Pr: Prandtl number

$\mathrm{Nu}_{h}$ : Local Nusselt number

$\overline{\mathrm{Nu}}_{\mathrm{ave}}$ : Average Nusselt number on the heat source

Ra: Rayleigh number

$T: \quad$ Temperature $(\mathrm{K})$

$x, y$ : Cartesian coordinate

$X, Y$ : Dimensionless Cartesian coordinate

$\beta$ : $\quad$ Thermal expansion coefficient $\left(\mathrm{K}^{-1}\right)$

$\delta: \quad$ Dimensionless position of the heat source

$\varepsilon: \quad$ Dimensionless length of the heat source

$\zeta$ : Dimensionless height of the heat source

७: Dimensionless temperature

$\nu$ : Cinematic viscosity $\left(\mathrm{m}^{2} \mathrm{~s}^{-1}\right)$

$\rho: \quad$ Density $\left(\mathrm{kg} \mathrm{m}^{-3}\right)$

$c$ : Cold wall

$h$ : Hot wall

exp: Experimental

num: Numerical

rg: Right side of the hot strip

lf: Left side of the hot strip.

\section{References}

[1] R. A. V. Ramos and L. F. Milanez, "Numerical and experimental analysis of natural convection in cavity heated from below," in Proceedings of the 11th International Heat Transfer Conference (IHTC '98), vol. 3, Kyongju, Korea, August 1998.

[2] E. Ntibarufata, M. Hasnaoui, E. Bilgen, and P. Vasseur, "Natural convection in partitioned enclosures with localized heating," International Journal of Numerical Methods for Heat \& Fluid Flow, vol. 3, no. 2, pp. 133-143, 1993.

[3] A. Valencia and R. L. Frederick, "Heat transfer in square cavities with partially active vertical walls," International Journal of Heat and Mass Transfer, vol. 32, no. 8, pp. 1567-1574, 1989.

[4] O. Aydin and W.-J. Yang, "Natural convection in enclosures with localized heating from below and symmetrical cooling from sides," International Journal of Numerical Methods for Heat \& Fluid Flow, vol. 10, no. 5, pp. 518-529, 2000.

[5] G. Cesini, M. Paroncini, G. Cortella, and M. Manzan, "Natural convection from a horizontal cylinder in a rectangular cavity," International Journal of Heat and Mass Transfer, vol. 42, no. 10, pp. 1801-1811, 1999.

[6] M. Raffel, C. Willert, and J. Konipenhans, Particle Image Velocimetry, Springer, Berlin, Germany, 1998.

[7] Dynamic Studio Manual, "Dantec Dynamics A/S," Publication number: 9040U1081, 2006.

[8] Fluent User's Guide, release 6.2.16, Fluent Incorporated (200501-06).
[9] W. Hauf and U. Grigull, "Optical methods in heat transfer," in Advances in Heat Transfer, J. P. Harnett and T. F. Irvine Jr., Eds., vol. 6, pp. 133-366, Academic Press, New York, NY, USA, 1970. 

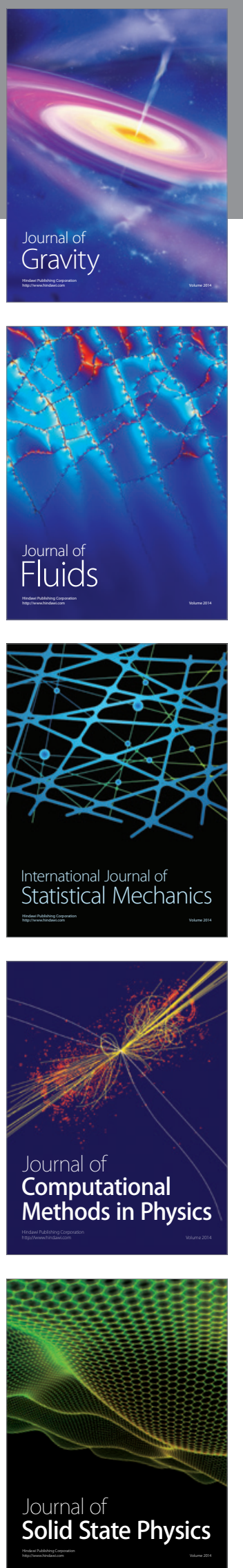

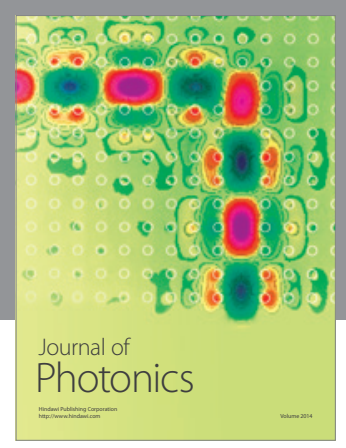

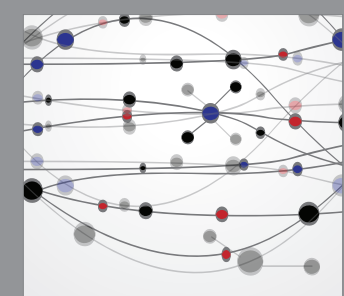

The Scientific World Journal
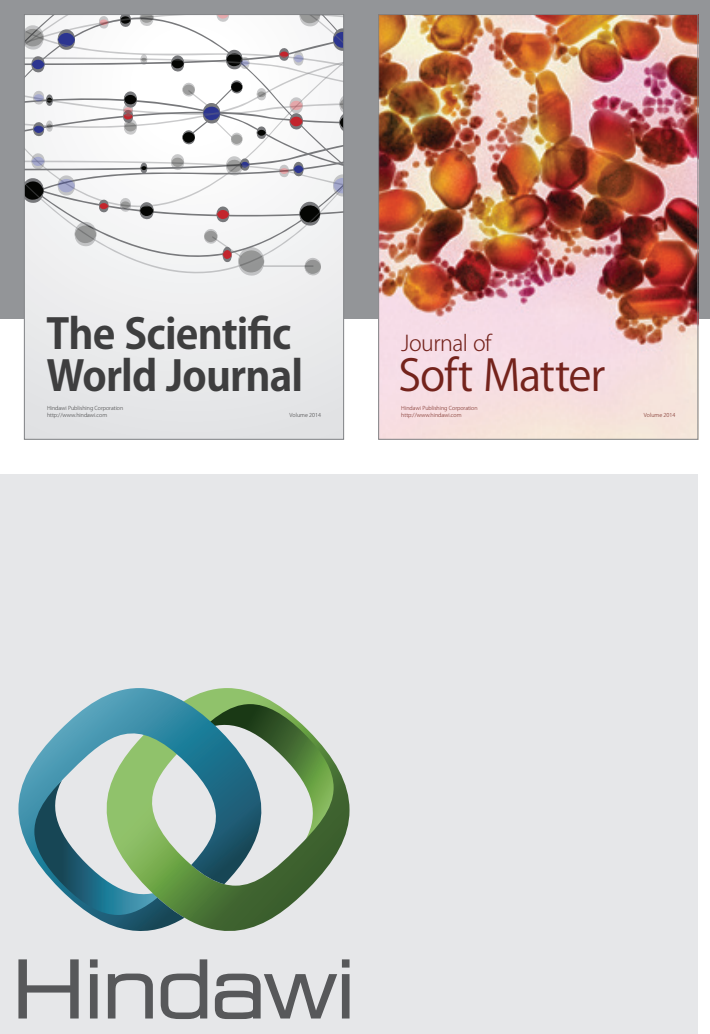

Submit your manuscripts at

http://www.hindawi.com
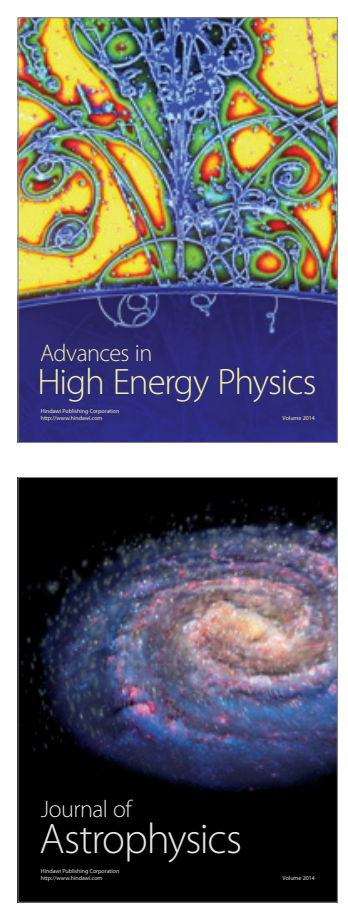
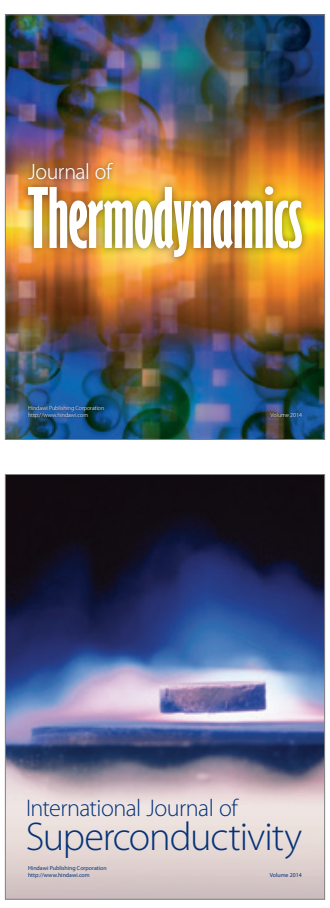
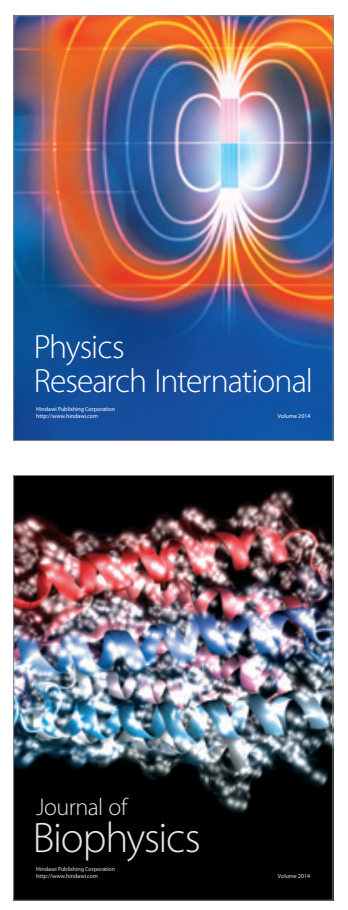
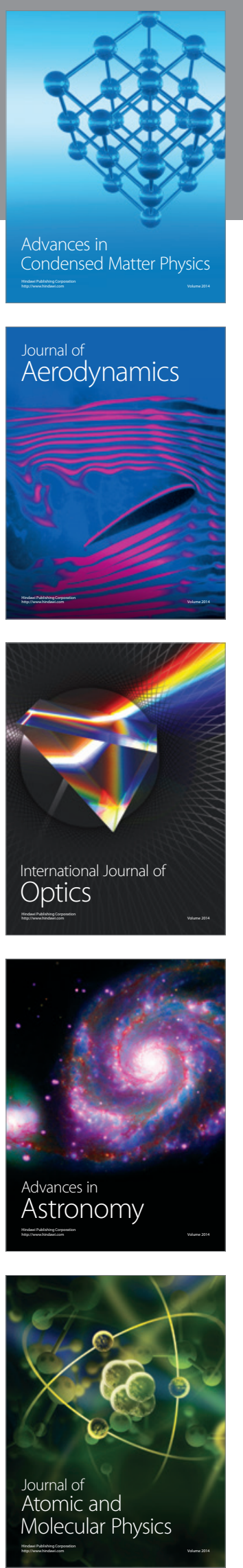